\title{
Influence of Solidity on Vertical Axis Wind Turbines
}

\author{
Teresa Parra ${ }^{* 1}$, Cristobal Uzarraga ${ }^{2}$, Armando Gallegos $^{3}$, Francisco Castro ${ }^{1}$
}

Accepted $15^{\text {th }}$ August 2014

DOI: $10.18100 /$ ijamec.42848

\begin{abstract}
This paper aims to predict the performance of a VAWT. The H-type Darrieus turbine consists of three straight blades with shape of aerofoil attached to a rotating vertical shaft. Bearing in mind the overall flow is characterized by important secondary flows, the turbulence model selected was realizable k-epsilon with non-equilibrium wall functions. Conservation equations were solved with a Third-Order MUSCL scheme using SIMPLE to couple continuity and momentum equations. A parametric study has been carried out to analyse the solidity effect on the non-dimensional curves so that the range of tip speed ratio of operation could be predicted as well as their self-starting behaviour.
\end{abstract}

Keywords: VAWT, CFD, solidity, efficiency

\section{Introduction}

One advantage of vertical-axis wind turbines (VAWT) is they do not need to be oriented in the wind direction however they operate at low tip speed ratios. Then the application is limited to small consumes. This paper aims to predict the performance of VAWT. The H-type Darrieus turbine consists of three straight blades with shape of aerofoil attached to a rotating vertical shaft. The criterion on the selection of this kind of turbines, despite its reduced efficiency, is the easy manufacture in workshops.

A parametric study has been carried out to analyze the camber effect on the non dimensional curves so that the self starting features [1] as well as the range of tip speed ratio of operation could be predicted. [2] analyzed the flow on the inner blade surface of an H-Darrieus vertical axis wind turbine with both straight and canted blades and different pitch angles. The work evidences that the reduction of solidity increases the range tip speed ratio.

\section{Numerical Model}

The general purpose code Fluent ${ }^{\circledR}$ v. 6.2 .16 was used to carry out the simulations. The governing continuity, momentum and turbulence equations were solved using a finite volume method. The set of equations are solved using a segregated solver.

Bearing in mind the computational cost and discretization errors, the best performance was that of the realizable $k-\varepsilon$. The near wall turbulence treatment was that of non-equilibrium wall functions because the overall flow is characterized by important secondary flows. Conservation equations were solved with a Third-Order MUSCL scheme using SIMPLE to couple continuity and

\footnotetext{
${ }^{1}$ Department of Energy and Fluid Mechanics, University of Valladolid, Spain

*Corresponding Author: Email: terpar@eii.uva.es

${ }^{2}$ Division of Mechatronic and Renewable Energies, Technological

University of Durango, Durango, Mexico

${ }^{3}$ Dept. of Mechanics, Guanajuato University Salamanca, Mexico

Note: This paper has been presented at the International Conference on Advanced Technology \& Sciences (ICAT'14) held in Antalya (Turkey), August 12-15, 2014.
}

momentum equations.

During VAWT operation, the performance depends mainly on the relative motion of the rotating blade fields and has a fundamental period which depends both on the rate of rotation and the number of blade passage. The transient study is necessary to characterise the hysteresis phenomenon. For the proposed calculations, the temporal discretization was achieved by imposing a physical time step equal to the lapse of time the rotor takes to make a $1.2^{\circ}$ rotation. Hence, more than three revolutions were carried out to prevent the influence of the initial conditions.

The mesh has two fluid volumes, one for the rotor where the moving mesh utility is used to establish the rotation velocity [3]. The other is the environment of the rotor. Different structured meshes were built. The 2D model has nearly 300 thousand hexahedral cells for a scaled model with chord $5 \mathrm{~cm}$, see figure 1 and table 1 for details.

Table 1. Details of the H-Darrieus

\begin{tabular}{|c|c|}
\hline Airfoil & NACA0025 \\
\hline Shaft radius & $0,01 \mathrm{~m}$ \\
\hline Rotor radius (R) & $0,0716 \mathrm{~m}$ \\
\hline Rotor Length (b) & $0,2 \mathrm{~m}$ \\
\hline Chord (c) & $0,05 \mathrm{~m}$ \\
\hline Rotor Area (A) & $0,02864 \mathrm{~m}^{2}$ \\
\hline Number of foils (N) & 3 \\
\hline Solidity $(\sigma)$ & 1,047 \\
\hline
\end{tabular}

Non dimensional parameters to be used are solidity ec. 1, Tip Speed Ratio ec. 2, momentum and power coefficients ecuations 3 and 4 respectively.

$$
\begin{aligned}
& \sigma=\frac{Z \cdot c \cdot b}{2 \cdot r \cdot b} \\
& T S R=\frac{\omega \cdot r}{v_{\infty}} \\
& C_{m}=\frac{T}{\frac{1}{2} \cdot \rho \cdot v_{\infty}^{2} \cdot r \cdot c \cdot b} \\
& C_{p}=\frac{T \cdot \omega}{\frac{1}{2} \cdot \rho \cdot v_{\infty}^{3} \cdot c \cdot b}
\end{aligned}
$$

Power coefficient is the product of momentum coefficient and the TSR. 


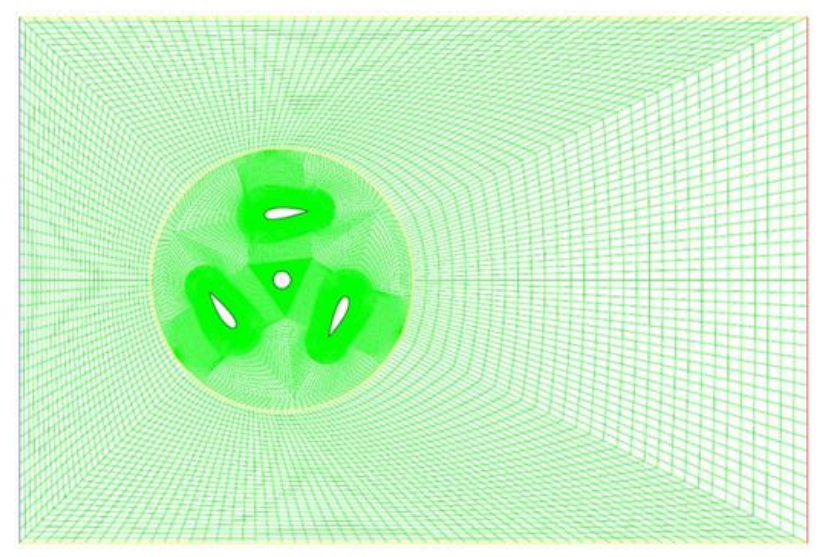

Figure 1. Mesh details

\section{Numerical Results}

Because of transient flow, results change over time as well as the torque on the shaft, there are time steps with high values of momentum coefficients, which correspond to the favorable positions of the wind turbine, and similarly there are times with torque close to zero or even negative, corresponding to unfavourable positions, see Fig. 2.

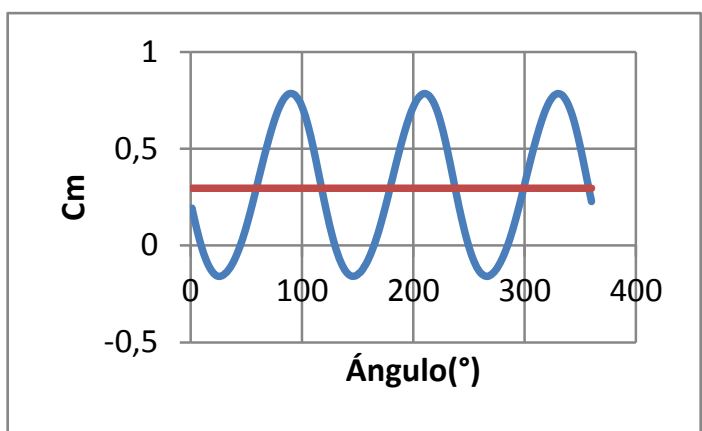

Figure 2. Instantaneous momentum coefficient at peak power conditions on no-symmetric NACA for an incident wind speed of $7 \mathrm{~m} / \mathrm{s}$ and solidity 1

The averaged momentum coefficient over one revolution of the rotor multiplied by the tip speed ratio produces the averaged power coefficient and it is one point of the characteristic curve of the wind turbine. Fig. 3 depicts a graph with the variation of $\mathrm{Cp}$ versus the TSR for two solidity values. The decrease of solidity produces an increase of the efficiency. Design conditions are those of maximum power coefficient. Also is desirable to work at higher TSR in order to produce electricity.

Fig. 4 let contrast the wakes in the most unfavourable and favourable positions of the airfoils. The weak wake corresponds with the maximum instantaneous power coefficient. Also the wake is important to establish distribution of turbines in a wind farm [4].

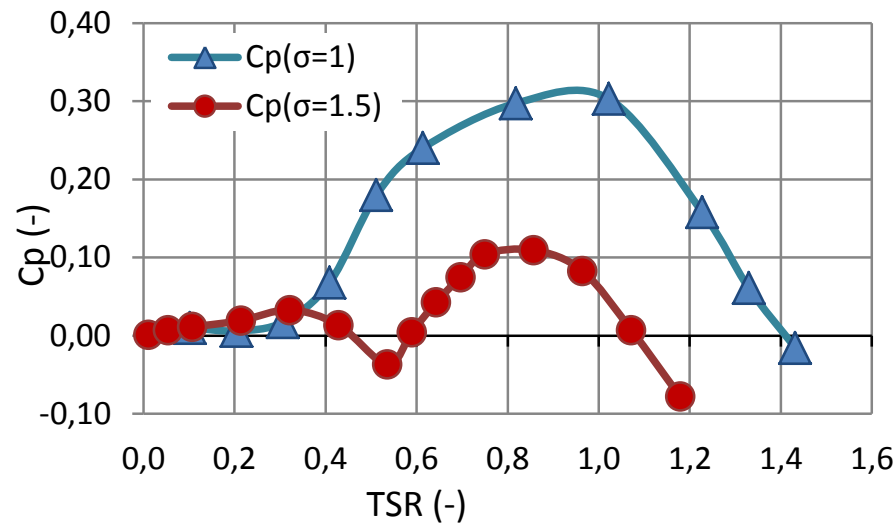

Figure 3. Averaged power coefficient versus the Tip Speed Ratio on nosymmetric NACA 0025 for an incident wind speed of $7 \mathrm{~m} / \mathrm{s}$ and solidity 1.5 and 1.
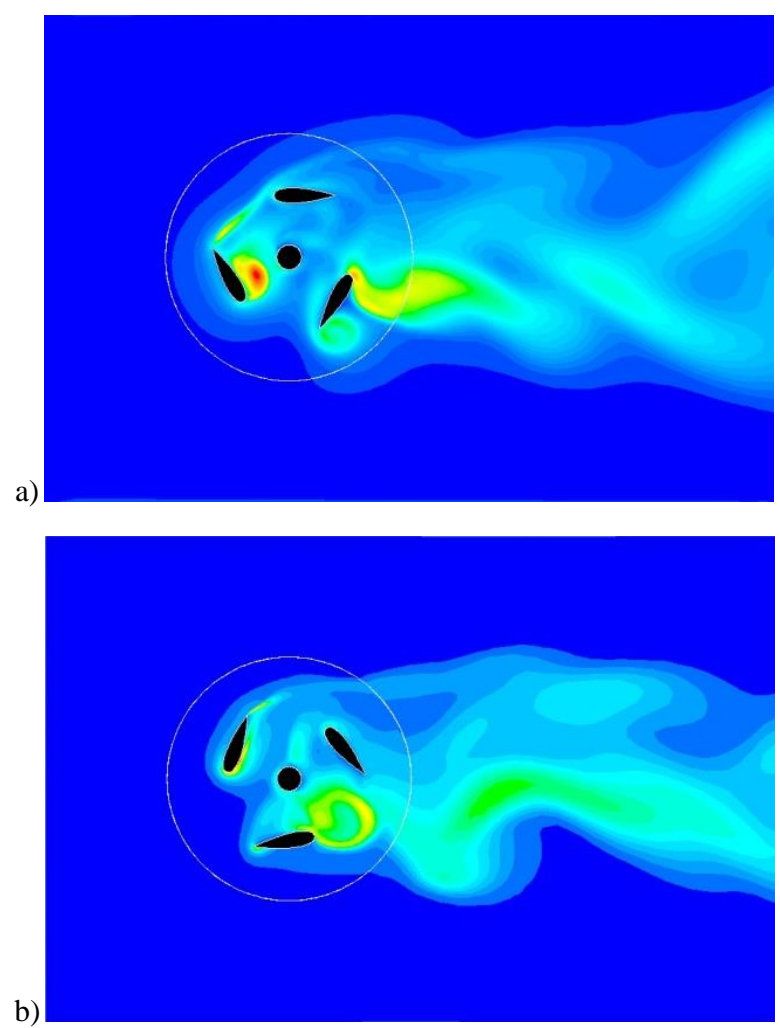

Figure 4. Contours of turbulent kinetic energy NACA 0025 for an incident wind speed of $7 \mathrm{~m} / \mathrm{s}$ and solidity 1.5 at design conditions. a) at minimum instantaneous power coefficient $b$ ) at maximum instantaneous power coefficient

Fig. 5 shows pressure contours at the most unfavourable (a) and favourable (b) position whereas fig. 6 shows velocity contours at the most unfavourable (a) and favourable (b) positions. "A" profile shows a stagnation point on the leading edge where the pressure is greater, fig. 5a. At this point, the speed is practically zero. One can also appreciate that the pressure in the outer area of the profile is larger (intrados) than the inner zone (extrados), although the difference is not quite decisive. Note in fig. $6 \mathrm{a}$ that the wake of "B" profile directly affects the leading edge of the "A" profile, so this is one of the possible causes of this position showing worse performance of the wind turbine. The force of the fluid on the profile "A" opposes the rotation of the turbine. 
a)

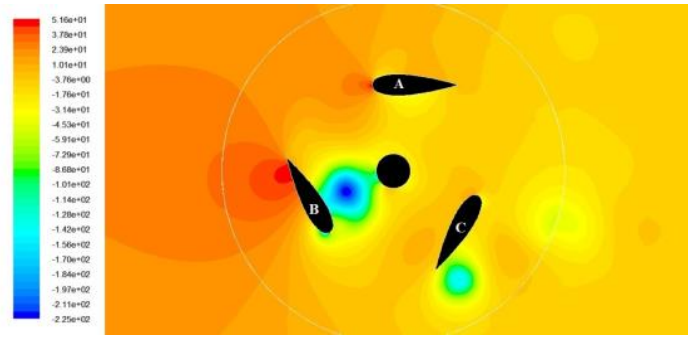

b)

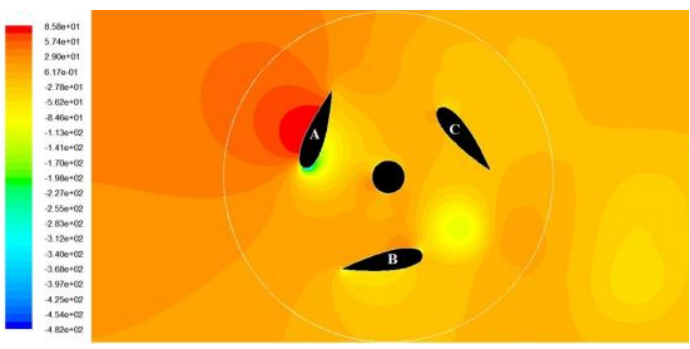

Figure 5. Contours of pressure NACA 0025 for an incident wind speed of $7 \mathrm{~m} / \mathrm{s}$ and solidity 1.5 at design conditions at maximum instantaneous power coefficient a) at minimum instantaneous power coefficient $b$ ) at maximum instantaneous power coefficient

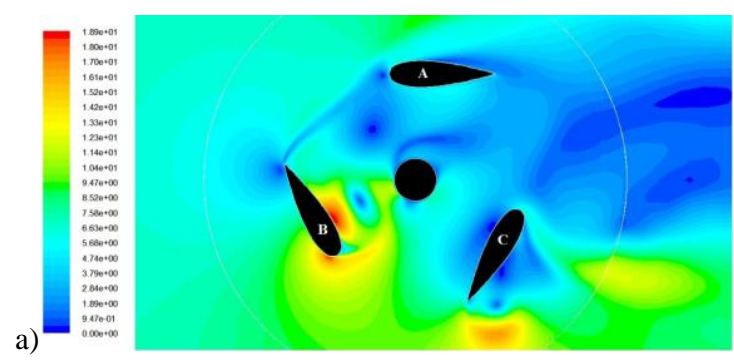

b)

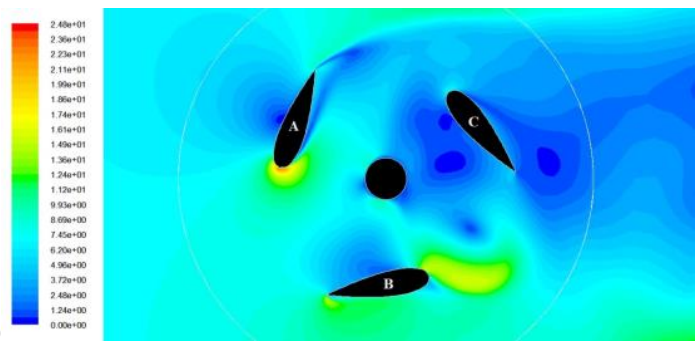

Figure 6. Contours of velocity NACA 0025 for an incident wind speed of $7 \mathrm{~m} / \mathrm{s}$ and solidity 1.5 at design conditions at maximum instantaneous power coefficient a) at minimum instantaneous power coefficient $b$ ) at maximum instantaneous power coefficient
It can be seen in fig. 5a, the outer part of the "B" profile corresponding to a region of high pressure (intrados), and that on the inner face of the profile has a high speed and a zone of low pressure (extrados). Moreover, the generation of a vortex near the profile is observed. The force exerted by the fluid on the "B" profile helps the rotation of the wind turbine.

Bearing in mind fig. $5 \mathrm{~b}$ and $6 \mathrm{~b}$, the " $\mathrm{A}$ " profile has high pressure at the outer edge producing thrust helping the rotation of the turbine. "B" and "C" airfoils have intrados in the inner surface.

\section{Conclusions}

An H-Darrieus air turbine using NACA airfoils has been modelled using a 2D general purpose CFD code.

The parametric analysis studied the effect of solidity. The criteria are based on the minimum period of time with instantaneous negative power coefficient to prevent the suffering of bearings. Also, the minimum range of TSR with averaged negative power coefficient to help the self-starting. Decreasing solidity improves the self-starting of the wind turbine and increases the range of TSR with energy production.

\section{Acknowledgements}

The author thankfully acknowledges the Spanish Ministry of Science and Innovation for the financial resources in the framework of the project reference ENE2011-25468.

\section{References}

[1] Baker J. R. 1983 "Features to aid or enable self starting of fixed pitch low solidity vertical axis wind turbines". Journal of Wind Engineering and Industrial Aerodynamics. Vol. 15 pp.369-380.

[2] Shawn A., Fiedler A., Tullis S. 2012 "Flow separation on a high Reynolds number, high solidity vertical axis wind turbine with straight and canted blades and canted blades with fences" Renewable Energy, Vol. 41, pp. 13-22

[3] Uzarraga N.C., Gallegos A., Parra M.T., Beltran J.M. 2012 "Numerical Analysis of Airfoils used at VAWT", ASME ESFuelCell2012-91113

[4] Liu X., Dang Q., Xi G. 2008. "Performance improvement of a centrifugal fam by using CFD". Engineering Applications of Computational Fluid Mechanics Vol. 2 no. 2 pp. $130-140$ 